\title{
Effect of hue on Visual Comfort and Depth Perception of Stereoscopic Images
}

\author{
Zhibin Su ${ }^{1,2,3, a}$, Hui Ren ${ }^{1,2,3, b}$ and Zeqian Zhou ${ }^{1, c}$ \\ ${ }^{1}$ Faculty of Science and Technology, Communication University of China \\ ${ }^{2}$ Key Laboratory of Acoustic Visual Technology and Intelligent Control System, Ministry of Culture \\ ${ }^{3}$ Beijing Key Laboratory of Modern Entertainment Technology \\ asuben@cuc.edu.cn, brenhui@cuc.edu.cn, ${ }^{\text {'}} 572285331 @ q q . c o m$
}

\begin{abstract}
Keywords: Hue, stereoscopic images, visual comfort, depth perception
Abstract. This paper has investigated the effect of hue on visual comfort and depth perception through subjective assessment experiments with the stereoscopic images generated by computer. The evaluation results indicated that the variable of hue would significantly affect the viewing experience under different combinations of disparity magnitude and disparity type. Of all the colors, cool colors got higher scores in both evaluation of visual comfort and depth perception. In addition, cyan was most comfortable for observers. Green had the strongest sense of space for depth perception, and yellow got the lowest rating score of the two indicators. Our study could provide important experimental data for improving the stereoscopic image and video technology, which has certain value and practical significance.
\end{abstract}

\section{Introduction}

In the field of stereoscopic 3D image and video making, according to the commonly used display technology with polarizer or active shutter, researchers are more concerned about the disparity related factors, such as disparity magnitude, disparity type, disparity distribution and disparity gradient $[1,2,3]$. Among them, the disparity magnitude, which is considered to be the most importance factor to cause accommodation-convergence conflict and stereo perception, will directly affect the visual experience. However, when the relevant study was explored in depth, more and more researchers have found that some content related features will affect the image evaluation under different disparity conditions, too. These new features includes: image spatial[4], the factor of luminance[5], contrast[6], color information, scale and distribution of the main part[7]. For the color image, chrominance is the main stimuli resource for visual perception and one of the key factors for 3D effect. During the two-dimensional visual research, we have concluded that different color will affect the psychological response of "cold" or "warm", "light" or "heavy" and "near" or "far"[8]. Therefore, it can be inferred that stereoscopic images with different colors could increase or decrease the viewing experience to some extent. The basic color information consists of hue, lightness and saturation, and this paper mainly focuses on the factor of hue for visual experience. The other color related factors, such as lightness and saturation are set to be the same. For 3D subjective assessment, the main perception indicators always include visual comfort and stereo perception [9]. Visual comfort here represents as a comprehensive measure of physical and psychological feeling. The concept of stereo has two different aspects: depth perception and volume perception. This paper chose to study only the effect of depth perception and visual comfort through relevant subjective experiments.

\section{Experiment Materials}

The materials used in this experiment were generated by computer, with a simple round as the main object. Several representative colors were chosen from the HSL model, they were red, orange, yellow, green, cyan and blue, in separate angular degrees of 0,30,60,120,180 and 240, with the max value of saturation and lightness. Half of them were warm colors. The main object had five levels of disparity 
magnitude(30, 40, 50, 60, 70 arc min )and two types of crossed disparity and uncrossed disparity. Besides, we set the middle gray color rounds under the same disparity conditions for comparison.

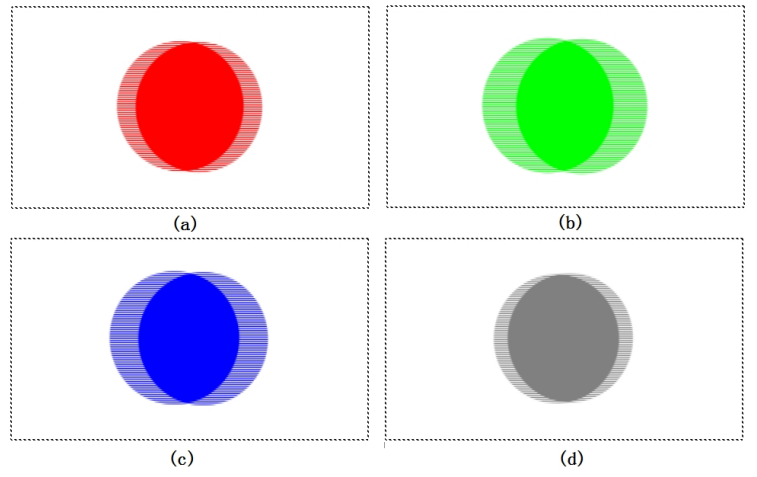

Figure 1 Evaluation materials

( a. Red, 40 arc min, uncrossed disparity; b. Green, 70 arc min, uncrossed disparity ;

c. Blue, 60 arc min; crossed disparity; d. Gray, 30 arc min, crossed disparity. )

Finally, the combinations of these three kinds of factor (disparity magnitude, disparity type and hue) generated 70 sequences with still images, which are shown in Fig. 1.

\section{Evaluation Method}

A modified version of Single Stimulus Continuous Quality Evaluation (SSCQE) method was used for the rating. More than twenty-five students participated in it and twenty of them provided effective and stable rating scores, including eleven males and nine females with a mean age of 22 years. All of them were not experts of stereo video and proved to have normal stereopsis by passing relevant stereo-acuity and multi-stereograph tests. Table 1 and 2 shows the detailed description of the rating in 5-point scales for visual comfort and depth perception. Because the test materials are only round objects without volume, we asked them to evaluate the sense of space they felt for depth perception.

Table 1 Evaluation scale of visual comfort

\begin{tabular}{|c|c|c|}
\hline \multicolumn{2}{|r|}{ Description of the Scale } & Score \\
\hline Very Comfortable & $\begin{array}{l}\text { The viewing experience is in complete accord with normal human } \\
\text { eye; comfortable and perfect }\end{array}$ & 5 \\
\hline Comfortable & The viewing experience is familiar with human eye, no discomfort & 4 \\
\hline $\begin{array}{l}\text { Perceptibly Uncomfortable but } \\
\text { acceptable }\end{array}$ & $\begin{array}{l}\text { The critical point that causes discomfort feeling. ( scores fall in this } \\
\text { range should be pay attention ) }\end{array}$ & 3 \\
\hline $\begin{array}{l}\text { Clearly Uncomfortable and } \\
\text { Unacceptable }\end{array}$ & $\begin{array}{l}\text { The critical point that causes discomfort and unacceptable feeling. ( } \\
\text { scores fall in this range should be avoided ) }\end{array}$ & 2 \\
\hline $\begin{array}{l}\text { Extremely Uncomfortable and } \\
\text { Extremely Unacceptable }\end{array}$ & --------- & 1 \\
\hline
\end{tabular}

Table 2 Evaluation scale of depth perception

\begin{tabular}{|c|l|c|}
\hline \multicolumn{2}{|c|}{ Description of the Scale } & Score \\
\hline Excellent & The sense of space is perfect. & 5 \\
\hline Good & $\begin{array}{l}\text { The sense of space is obvious, the distance between main object and screen can be } \\
\text { recognized clearly. }\end{array}$ & 4 \\
\hline Fair & The sense of space can be perceived, but it is not obvious. & 3 \\
\hline Poor & $\begin{array}{l}\text { The sense of space can be perceived slightly but inconspicuous. } \\
\text { (You might think the main object is almost on the screen sometimes) }\end{array}$ & 2 \\
\hline Bad & $\begin{array}{l}\text { Without any sense of space or 3D perception, the main object looks like just on the } \\
\text { screen }\end{array}$ & 1 \\
\hline
\end{tabular}

The experiment environment had followed the demand of subjective assessment method of image quality of 3DTV[9]. The format of test sequences were $1920 * 1080$, side-by-side (half). The same 
sequence occurred twice in randomized orders. Observers watched the arranged sequences with polarized glasses and the professional LCD monitor of SONY 2451TD at the distance of $1.8 \mathrm{~m}$.

\section{Results}

The analysis of variance (ANOVA) was performed for various combinations of the three factors for visual comfort and depth perception separately. For the assessment of visual comfort, the factor of hue was statistically significant, $F(5,95)=23.530, \mathrm{p}<0.01$. The interactions of disparity $\times$ hue, disparity type Xhue were both significant. For disparity Xhue, $\mathrm{F}(20,380)=2.803$, $\mathrm{p}<0.01$; for disparity type $X$ hue, $\mathrm{F}(5$, $95)=2.884, p<0.05$. The triple interaction of disparity type $x$ disparity $x$ hue was also significant, $F(20$, $380)=2.430, \mathrm{p}<0.01$.

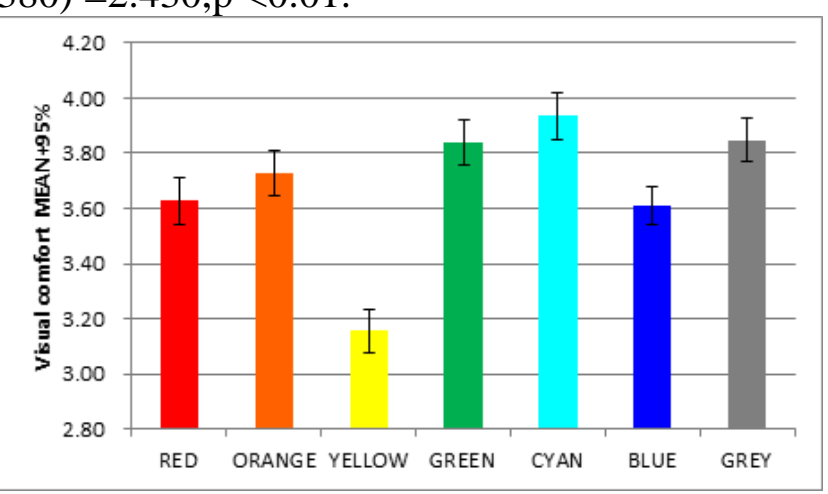

Figure 2 Evaluation results of visual comfort

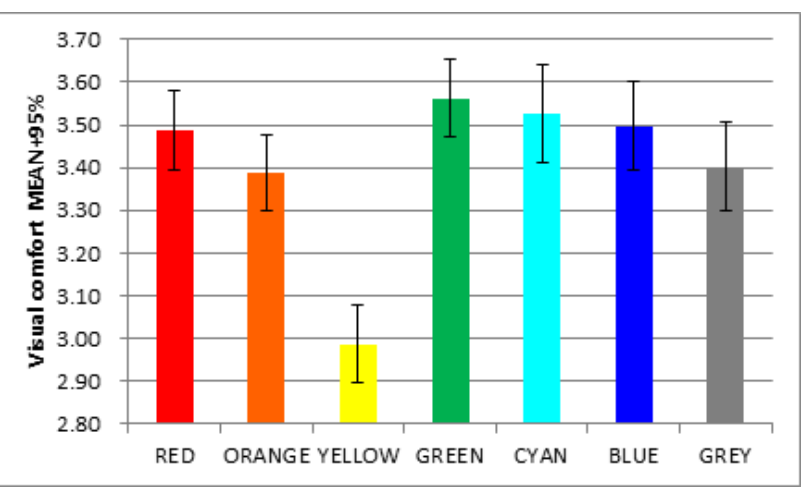

Figure 3 Evaluation results of depth perception

Figure 2 shows the main rating results for visual comfort. Of all the six colors, cyan was most comfortable under different combinations of disparity, its average rating score had exceeded 3.8, which was quite closed to the scale of comfort. The most uncomfortable color was yellow, which was lower than 3.2. In addition, the cool colors got higher scores than the warm ones. Through the comparison of neutral color, grey was more comfortable than the other colors except cyan. When we only consider the disparity of 7 arc min, under positive condition, green had the highest rating score of 4.18, which means it was comfortable even the disparity magnitude had exceed the comfortable fusion region; under negative condition, cyan was the most comfortable color, with the rating score of 3.52, while the other five colors were all under 3.4. In both conditions of disparity type, yellow got the lowest score, which was close to the scare of unacceptable feeling. Considering that region contrast was one of the factors to affect visual comfort for grayscale image, we tried to find the same relationship for these materials. However, for the color of yellow, its lowest region contrast didn't improve the visual comfort. The green had the highest rating score, with its region contrast lower than orange, yellow and blue. This was different from the effect of grayscale image.

During the assessment of depth perception, the factor of hue was also significant, $F(5,95)=6.709$, $\mathrm{p}<0.01$. The interactions of disparity $\times$ hue was significant, $\mathrm{F}(20,380)=2.442, \mathrm{p}<0.01$. For the triple interaction of disparity typexdisparity $\times$ hue, $\mathrm{F}(20,380)=1.621, \mathrm{p}<0.05$. The evaluation results were shown in figure 3 . The green got the highest score for depth perception, which was above 3.5; while the score of cyan and blue was a little lower than it. The rating score of yellow was less than 3.0, which means its performance was poor in depth. All of the cool colors got higher scores than the three warm colors. For the warm colors, none of them exceeded the scale of 4.0(obvious sense of space); for the cool colors, the highest score of green got 4.04 at the disparity of 50 arc min, the highest score of cyan got 4.02 at 60 arc min. The depth experience of grey was weaker than the three cool colors of green, cyan and blue. It was known that the feeling of depth would be higher with the increasing of disparity. However, not all colors could get highest rating score at the largest magnitude of disparity. For the 
color of red, cyan and green, the strongest sense of depth would be felt at the disparity of 60 arc min or 50 arc min. It can be inferred that for those colors, depth feeling might decrease when the image was not so comfortable to watch. When the disparity magnitude was at 30 arc min, among all of the colors, Cyan got the highest score of 3.31 (under positive disparity) and 3.63(under negative disparity), while yellow got both the lowest score of 2.66 and 2.80 .

\section{Conclusions}

In this study, we had investigated the relationship between the color information of hue and two common indicators for viewing experience: visual comfort and depth perception. The subject evaluation results confirmed that hue was a significant variable that could affect both the indicators. For visual comfort, cyan was the most comfortable color under different combinations of disparity. For the depth perception, green provided the best sense of space for the observers. In brief, the cool colors got higher rating scores than the warm ones; yellow got both the lowest rating score for the two indicators. One of its practical values was, when the disparity of the main object was too large or small, if it was possible, we could control the display effect by adjusting its color. On the contrary, if the color of the main object was settled, we should control its disparity according to the fusion region and its content feature of hue.

Overall, to the research of stereoscopic images, there had been a lot of experimental data for visual comfort during the past twenty years; however, less work was done for the accurate feeling of depth perception. Our research has considered both the two aspects for watching demand and proved that color information was a special kind of psychological and physiological factors to affect the visual comfort and depth perception, which is helpful for the further study of objective model construction and acquisition technology for stereoscopic images and videos.

\section{Acknowledgements}

This work was financially supported by the Engineering Research Project of Communication University of China, Study on stereoscopic image perception and subjective evaluation based on content and disparity information. (Project No.3132016XNG1622)

\section{References}

[1] Tam W.J, Speranza. F, Yano. S, Shimono. K, Ono. H. Stereoscopic 3D-TV: Visual Comfort[J]. Broadcasting, IEEE Transactions on.,2011,57,(2),335-346.

[2] Lambooij M, IJsselsteijn W A, Heynderickx I. Visual discomfort of 3D TV: assessment methods and modeling[J]. Displays, 2011, 32(4): 209-218.

[3] Jiang Q; Shao F; Jiang G , et al , an objective stereroscopic image visual comfort assessment metric based on visual important regions[J], Journal of Electronics \& Information Technology, 2014,04

[4] Okada Y, Ukai K, Wolffsohn J. Target spatial frequency determines the response to conflicting defocus-and convergence-driven accommodative stimuli[J]. Vision research, 2006, 46, (4), pp. 475-484.

[5] Cho S.H, Kang H.B. Visual discomfort under various brightness conditions using eye movements in watching stereoscopic 3D video[J]. Proceedings of SPIE - The International Society for Optical Engineering, 2014, 9011(12):2978-2982 
[6] Ren H, Su Z.B, Lv C.H, et al. Effect of region contrast on visual comfort of stereoscopic images[J]. Electronics Letters, 2015, 51(13): 983-985.

[7] Su Z.B, Zou F.J, Ren H. Effect of main part size and disparity distribution on visual comfort of Stereoscopic images[C]. MEITA 2015, Yingchuan, China,2015.08.

[8] . Lin Z.X, Psychology of color vision[M], China Renmin University Press, 2011.06

[9] ITU-R. Subjective assessment of stereoscopic television pictures[S]. ITU-R Recommendation BT.1438, 2000. 